\title{
Cardiovascular effects of dexamethasone in the preterm infant
}

\author{
Nick Evans
}

\begin{abstract}
Twenty preterm infants were studied serially with Doppler echocardiography to document changes in pulmonary artery pressure, myocardial thickness, and patent ductus arteriosus associated with dexamethasone treatment for chronic lung disease. Pulmonary artery pressure was assessed with Doppler, by its inverse correlation with the ratio of the pulmonary artery time to peak velocity (TPV) and right ventricular ejection time (RVET).

Eleven of the 20 infants showed an increase in TPV/RVET after commencing steroids, suggesting a fall in pulmonary artery pressure. This change was not sustained in most cases and had no correlation with the improvement in respiratory status. Ventricular septal and left ventricular posterior wall thickness increased in all 11 infants in whom it was measured. The median increase was respectively 0.9 and $0.8 \mathrm{~mm}$. In most infants this increase was small, less than $1 \mathrm{~mm}$, however two infants developed marked septal hypertrophy with Doppler evidence of left ventricular outflow tract obstruction. Dexamethasone had no consistent closing effect on a patent ductus arteriosus in four infants.
\end{abstract}

Myocardial hypertrophy occurs in most infants, in some it is severe. It would seem prudent to monitor echocardiographically for this side effect.

(Arch Dis Child 1994; 70: F25-F30)

This paper draws together the results of serial Doppler echocardiography on preterm infants receiving dexamethasone for treatment of chronic lung disease. The aim was to document the effect of this drug on three facets of cardiovascular structure and function, namely pulmonary artery pressure, myocardial hypertrophy, and patent ductus arteriosus.

The mechanism by which dexamethasone causes an immediate improvement in respiratory and oxygen requirements is unclear, although there is evidence that the effect is mediated through the suppression of inflammation. ${ }^{1}$ Part of this anti-inflammatory effect results in a global inhibition of prostaglandin synthesis. ${ }^{23}$ In the lungs these prostaglandins may have a vasoactive role, both dilator and constrictor. ${ }^{4}$ Pulmonary artery pressure is usually high in infants with chronic lung disease. ${ }^{5}$ In this study, the aim was to assess whether changes in pulmonary artery pressure might relate to some of the improvement associated with dexamethasone.

In addition, this study examines in more detail two previously documented cardiac side effects of dexamethasone in an unselected group of infants. Firstly myocardial hypertrophy ${ }^{6}$ : the clinical significance of this is unclear, although the report of Werner et al suggested that it occurred in the majority of cases. ${ }^{6}$ Recently three cases were reported where significant left ventricular outflow tract obstruction and cardiac failure occurred as a result of steroid induced hypertrophy. ${ }^{7}$ In this study I have measured changes in left ventricular myocardial thickness to document the incidence, severity, and clinical significance of this side effect. Secondly patent ductus arteriosus closure: a further report related four cases where there was a temporal association between commencing dexamethasone and clinically assessed closure of a patent ductus arteriosus. ${ }^{8}$ I have documented, with serial echocardiography, ductal status in those infants with a patent ductus arteriosus when dexamethasone was commenced, to see if there was a consistent effect on patency.

\section{Methods}

Twenty preterm infants were studied with serial Doppler echocardiography before, during, and after receiving dexamethasone for chronic lung disease. They had a mean birth weight of $817 \mathrm{~g}$ (range 519-1425 g) and a mean gestation of 26 weeks (range 23-30 weeks). The median postnatal age at the time treatment commenced was 23.5 days with a range from 7-96 days. Nine infants were studied at the John Radcliffe Maternity Hospital, Oxford between February and August 1991. Eleven infants were studied at King George V Hospital, Sydney between February and December 1992. Only one infant who received dexamethasone during these time periods was not studied. The investigator was on leave when that infant was treated.

Pulmonary artery pressure was assessed by pulsed Doppler recording of pulmonary artery flow velocity using its inverse correlation with the ratio of the time to peak velocity (TPV) and the right ventricular ejection time (RVET). ${ }^{910}$ In Oxford, the infants were studied with an Ultramark 4 scanner using a $7.5 \mathrm{MHz}$ probe which incorporated a $5 \mathrm{MHz}$ pulsed Doppler crystal. The pulmonary artery was imaged from the parasternal long axis view and the pulsed Doppler range gate was 
placed distal to the pulmonary valve. Care was taken to ensure the Doppler velocity time signal was recorded from laminar, non-turbulent flow in the centre of the pulmonary artery. ${ }^{11}$ The signal was recorded and frozen on screen. Using the incorporated measurement calipers, TPV and the RVET were measured, a mean of three measurements was taken and then expressed as the ratio TPV/RVET. The TPV was defined as the time interval between the velocity time signal leaving the baseline and reaching its peak velocity and the RVET as the time between the signal leaving and returning to the baseline. The ductus arteriosus was imaged from a high parasternal view, and a pulsed Doppler search of the pulmonary end of the duct was made for ductal shunting. Where the duct was patent, the ratio of the left atrial diameter to the aortic root (LA:AO) was also measured using a standard method. ${ }^{12}$

In Sydney, the infants were studied with an Accuson 128XP/10 scanner with colour flow Doppler mapping. TPV and RVET were measured as in Oxford except the velocity time signal was recorded on videotape and a mean of five measurements were taken from the videotape. Colour flow Doppler mapping was used to assess for ductal shunting. The duct was classified as closed when it was not patent on two dimensional imaging and no shunt could be detected on pulsed Doppler and or colour flow assessment. In addition, in these 11 infants, measurements of end diastolic interventricular septal thickness (EDIVS) and left ventricular posterior wall (EDLVPW) thickness were taken from left ventricular $M$ mode echocardiograms using a standard method. ${ }^{12}$ The reported measurements were taken from videotapes and were averaged from three cardiac cycles. If there was marked septal hypertrophy and evidence of turbulence in the left ventricular outflow tract (LVOT) on colour flow Doppler mapping, pulsed Doppler assessment of the left ventricular outflow tract was performed from an apical view modified to incorporate the whole outflow tract including the ascending aorta.

Infants were studied in the 24 hours before receiving the first dose of dexamethasone. Echocardiograms were repeated alternate daily for the first week of treatment and then twice weekly until the week after dexamethasone was stopped.

Dexamethasone was given by different protocols in the two centres. In Oxford, the regimen was $0.6 \mathrm{mg} / \mathrm{kg}$ for seven days, 0.4 $\mathrm{mg} / \mathrm{kg}$ for four days, and $0.2 \mathrm{mg} / \mathrm{kg}$ for four days. In Sydney, the regimen was $0.5 \mathrm{mg} / \mathrm{kg}$ for three days, $0.3 \mathrm{mg} / \mathrm{kg} /$ day for three days, and then a dose reduction by $10 \%$ every three days until $0.1 \mathrm{mg} / \mathrm{kg}$ was reached. The last week of the course was given as $0 \cdot 1 \mathrm{mg} / \mathrm{kg}$ on alternate days.

At the time of each echocardiogram, clinical data on ventilatory requirements was collected. This included inspired oxygen concentration $\left(\mathrm{FIO}_{2}\right)$, peak inspiratory pressure, and ventilator rate. These were combined to produce an arbitrary index of ventilator and oxygen

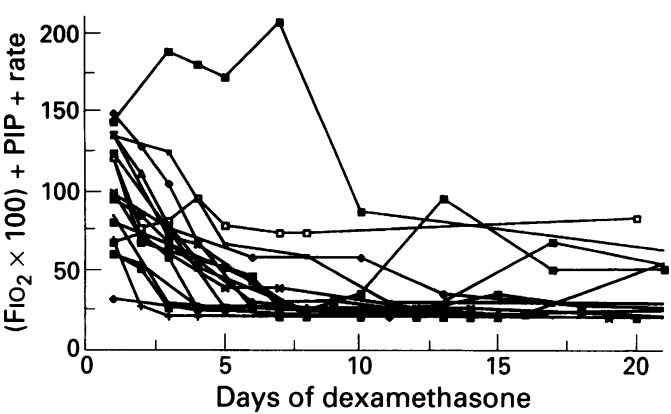

Figure 1 Change in the score of ventilation and oxygenation after treatment with dexamethasone in 18 of the 20 infants; PIP = peak inspiratory pressure.

requirements from the formula $\left(\mathrm{FIO}_{2} \times\right.$ 100) + peak inspiratory pressure+ventilator rate. If the infant was receiving nasal continuous positive airway pressure (CPAP), 5 was added to the percentage oxygen. If the infant was in a headbox the index just represented the percentage oxygen. Thus an infant in air would have a score of 21 .

The study was approved by both hospital ethical committees. Statistical analysis was by $\chi^{2}$ and unpaired $t$ test with statistical significance accepted at $p$ values of less than 0.05.

\section{Results}

(1) CHANGE IN VENTILATOR AND OXYGEN REQUIREMENTS

All except one infant was being ventilated at the time the dexamethasone was commenced. The mean oxygen and ventilation score at this time was 100 with a range from 32-148. All except one infant showed an improvement in ventilation and oxygen requirements in the first three days after commencing dexamethasone. Figure 1 shows the change in the score with time in 18 of the infants. In the other two infants, complete data to produce the score were lost. These two infants were weaned from the ventilator respectively two and six days after commencing dexamethasone.

\section{(2) CHANGE IN TPV/RVET (PULMONARY ARTERY} PRESSURE)

The mean TPV/RVET before commencing dexamethasone was $0 \cdot 27$ (range $0 \cdot 18-0 \cdot 4$ ). The normal range for TPV/RVET in a group of preterm infants without respiratory

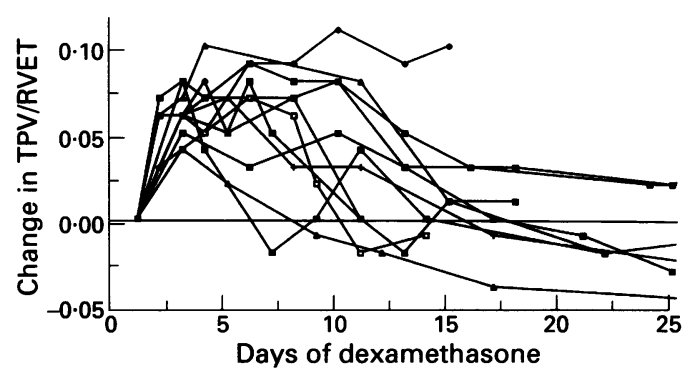

Figure 2 Change in TPVIRVET after treatment with dexamethasone in the 11 infants who demonstrated a consistent increase in TPVIRVET. 


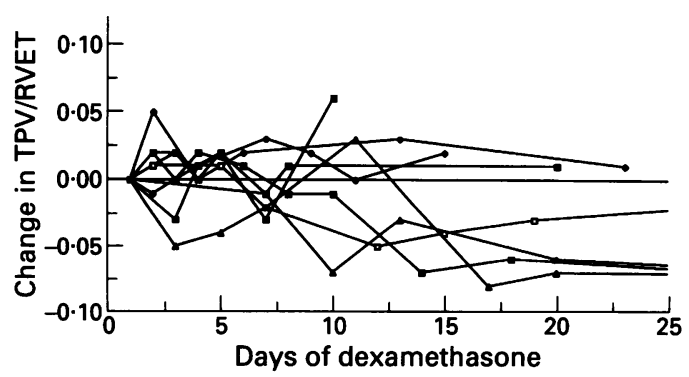

Figure 3 Change in TPV/RVET after treatment with dexamethasone in the nine infants who demonstrated no consistent increase in TPV/RVET.

problems was $0 \cdot 33-0 \cdot 41 . .^{13}$ Only three of the infants fell within this range before treatment, in the remainder a low TPV/RVET suggested raised pulmonary artery pressure. In 11 of the infants there was an increase in TPV/RVET of at least 0.02 which was sustained for at least two consecutive measurements in the first week of treatment (fig 2). In 10 of the infants this increase was not sustained and the TPV/RVET had returned towards the baseline by the end of the second week of treatment. After this time, five of the infants showed a trend to a further reduction in TPV/RVET. In one infant the increase was sustained until the last measurement which was the day after treatment was complete. In the other nine infants, there was no consistent increase in the ratio (fig 3). Four of the infants in this group showed a trend to a reduction in ratio, suggesting an increasing pulmonary artery pressure after the first week of treatment.

The group of infants who showed a sustained increase in TPV/RVET did not necessarily have the most marked improvement in respiratory status. There was no correlation between the maximum change in TPV/RVET in the first week of treatment and the change in ventilation and oxygen score at the same time. There was no significant difference between the two groups in birth weight, gestation, severity or length of acute respiratory illness, postnatal age at treatment, and TPV/RVET before treatment commencing (table). Six of nine $(66 \%)$ infants studied in Oxford showed a sustained increase in TPV/RVET compared with five of $11(45 \%)$ studied in Sydney, not a significant difference. In the first week of treatment, there was no significant change in heart rate at the time of study either in the group as a whole or analysing separately those

Comparison between the infants who did and did not show consistent increase in TPV/RVET. Figures are given as mean (SD); none of the differences are significant

\begin{tabular}{|c|c|c|}
\hline & $\begin{array}{l}\text { Increase } \\
\text { in TPV/RVET } \\
(n=11)\end{array}$ & $\begin{array}{l}\text { No increase } \\
\text { in TPV/RVET } \\
(n=9)\end{array}$ \\
\hline $\begin{array}{l}\text { Birth weight (g) } \\
\text { Gestation (weeks) } \\
\text { Postnatal age (days) } \\
\text { Maximum FIo } \\
\text { Maximum peak pressure }\end{array}$ & $\begin{array}{c}731(149) \\
25 \cdot 5(1 \cdot 3) \\
33 \cdot 3(27 \cdot 3) \\
0.69(0 \cdot 22)\end{array}$ & $\begin{array}{l}921(257) \\
26 \cdot 5(1 \cdot 8) \\
22 \cdot 1(10 \cdot 7) \\
0 \cdot 75(0 \cdot 26)\end{array}$ \\
\hline $\begin{array}{l}\left(\mathrm{cm} \mathrm{H} \mathrm{H}_{2} \mathrm{O}\right) \\
\text { Pretreatment TPV/RVET }\end{array}$ & $\begin{array}{l}20(9 \cdot 75) \\
0.26(0.07)\end{array}$ & $\begin{array}{l}21(13 \cdot 2) \\
0.28(0.03)\end{array}$ \\
\hline
\end{tabular}

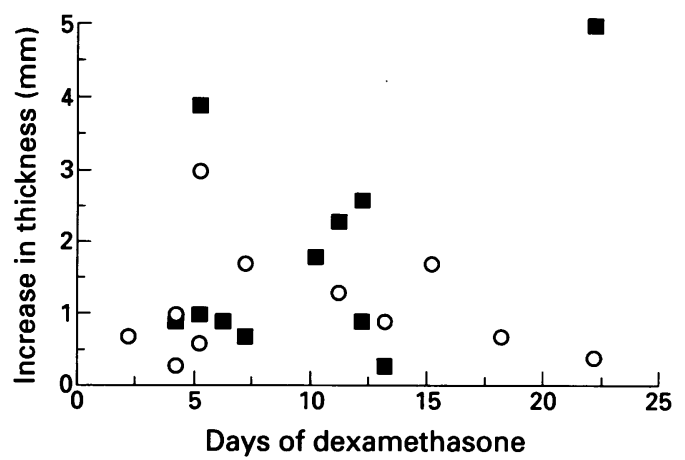

Figure 4 Individual maximum increases in EDIVS (closed squares) and EDLVPW (open circles), plotted on the day of dexamethasone treatment that the maximum increase was recorded.

that did and did not demonstrate an increase in TPV/RVET.

\section{(3) CHANGE IN LEFT VENTRICULAR MYOCARDIUM}

This was measured on the 11 infants studied in Sydney, so all these infants were treated with the prolonged dosage regimen. None of these infants had significant problems with glycaemic control and none had required treatment with insulin. Nine of the infants had been given inotropic support (dopamine or dobutamine) for low blood pressure or low cardiac output during the acute phase of their illness, but only one was still on inotropic support when steroids were commenced.

The mean EDIVS thickness before commencing treatment was $3.2 \mathrm{~mm}$ (range 2.3 $4.2 \mathrm{~mm}$ ). All 11 infants studied developed some increase in EDIVS thickness (fig 4). The median maximal increase was $0.9 \mathrm{~mm}$ (range $0.2-4.9 \mathrm{~mm}$ ), the timing of this maximal increase is shown for each infant in fig 4 . The degree and rate of this increase was very variable, six of the 11 infants had a maximal change in septal thickness of less than $1 \mathrm{~mm}$. The other five had an increase in EDIVS thickness of between 1.7 and $4.9 \mathrm{~mm}$. Two of these infants developed Doppler evidence of LVOT obstruction.

The first of these was an $850 \mathrm{~g}, 26$ week gestation second twin. This infant's respiratory status had improved by day 13 of treatment to requiring air with nasal CPAP. On day 14 , an increasing respiratory rate and oxygen requirement developed. A soft systolic murmur was noted at the upper sternal edge. Chest radiography showed a slight increase in opacification of both lung fields. Echocardiography at this time showed an EDIVS thickness of 5.6 mm with evidence of turbulence in the LVOT proximal to the aortic valve on colour flow mapping with a peak velocity in the LVOT of $1.2 \mathrm{~m} / \mathrm{sec}$. Treatment with intravenous frusemide was commenced and the infant was in air by the next day, regular frusemide was continued. By day 22, the EDIVS thickness was $7.9 \mathrm{~mm}$ and peak velocity in the LVOT was $1.37 \mathrm{~m} / \mathrm{sec}$. By day 28 the last day of treatment, the EDIVS was $7.9 \mathrm{~mm}$ and the peak velocity in the LVOT was $2.19 \mathrm{~m} / \mathrm{sec}$. Four 
days later, the EDIVS was $5 \cdot 3 \mathrm{~mm}$ and 10 days later $3.3 \mathrm{~mm}$ and there was no evidence of LVOT obstruction. This infant's twin was also treated with dexamethasone but did not show the same degree of hypertrophy.

The second of these was a $1425 \mathrm{~g}, 30$ week infant. This infant had a degree of septal hypertrophy before treatment, with a baseline EDIVS of $4.2 \mathrm{~mm}$. By day 10 of treatment this had increased to $5.9 \mathrm{~mm}$ and there was colour flow evidence of turbulence in the LVOT. Peak velocity in the LVOT was 1.55 $\mathrm{m} / \mathrm{sec}$. The infant was transferred out for surgery for a gastrointestinal complication, so further acute study was not possible. Ten days after steroids were stopped the EDIVS was $3 \cdot 8$ $\mathrm{mm}$ and there was no evidence of LVOT obstruction.

The mean EDLVPW before commencing treatment was $2.6 \mathrm{~mm}$ (range $1.7-3.5 \mathrm{~mm}$ ). All infants showed some increase in EDLVPW, the median maximal increase was $0.8 \mathrm{~mm}$ (range $0.2-2.9 \mathrm{~mm}$ ). Again the degree and rate of this increase was very variable (fig 4). Only four infants showed an increase of more than $1 \mathrm{~mm}$. There was no correlation between the increase in EDIVS and that in EDLVPW in an individual infant. In all infants the thickness of both the interventricular septum and the LVPW had returned to close to the baseline measurements when studied in the week after dexamethasone had stopped.

\section{(4) PATENT DUCTUS ARTERIOSUS}

Four infants had a patent ductus arteriosus at the time of commencing treatment. All were asymptomatic and small in size with local constriction on two dimensional imaging and or colour flow mapping. They had moderately raised LA:AO with a mean of 1.41 (range $1 \cdot 3-1 \cdot 5)$. In three of the infants the patent ductus arteriosus closed during the course of treatment. The following are respectively the day of treatment on which the patent ductus arteriosus was last seen patent and first seen closed for each of these three infants, that is, closure occurred at some time between these two days; days 24 and 28 , days 8 and 10 , days 4 and 6 . In the other infant closure did not take place during the time period of the study. In the three in which the duct closed, the timing of closure corresponded to respectively the eighth, fourth, and fifth postnatal week.

\section{Discussion}

Dexamethasone produces rapid improvements in ventilator and oxygen requirements in chronic lung disease. This acute effect has been well described in several randomised controlled trials ${ }^{14} 15$ and has been confirmed in this study; all except one infant showed an immediate improvement in respiratory status after commencing dexamethasone. The mechanism of this effect remains uncertain. It has been shown that there are significant early reductions in lung compliance and that at least some of this effect is probably mediated through the suppression of chronic inflammatory processes. 1 Part of this suppression of inflammation involves a general suppression of potentially vasoactive hormones such as prostaglandins. ${ }^{23}$ This study has confirmed that pulmonary vascular resistance is usually high in chronic lung disease. Seventeen of the 20 infants studied had a TPV/RVET below the normal range before commencing steroids. The original hypothesis of this study was that part of the effect of dexamethasone might be mediated through a reduction in pulmonary vascular resistance. The data did not support this hypothesis. Eleven of the 20 infants studied demonstrated an increase in TPV/RVET suggesting a fall in pulmonary artery pressure. The average change in TPV/RVET in this group was an increase of 0.06 . From the data of Kosturakis et al, ${ }^{10}$ this would suggest an average fall in mean pulmonary artery pressure of $8.75 \mathrm{~mm} \mathrm{Hg}$ in this group. However, two points from the data would suggest that this is not a primary effect associated with the improvement in respiratory status. Firstly, this change in TPV/RVET was only sustained in one infant while the improvement in respiratory status was maintained and secondly, the other nine infants showed no consistent change in TPV/RVET but demonstrated respiratory improvement. There was no correlation between change in TPV/RVET and respiratory improvement. Why did the TPV/RVET change only in 11 of the infants? I could find no clear delineating factors between the two groups. It seems that the changes demonstrated were independent of changes in lung function. Why were these changes in TPV/RVET not sustained? It may be that this was a dose related effect. The TPV/RVET tended to return to the baseline in the second week of treatment as the dose of dexamethasone dose was being reduced. It is also interesting that more of the infants on the higher initial dose regimen used in Oxford showed consistent changes in TPV/RVET.

The other non-invasive means of assessing pulmonary artery pressure is with the measurement of the peak velocity of a tricuspid incompetence jet. While this method is probably more accurate than TPV/RVET, tricuspid incompetence is not present in all infants even when pulmonary artery pressure appears to be high $^{16}$; this limits its usefulness in longitudinal studies such as this one. I chose to use the TPV/RVET ratio in this study as this is a measure which can be made in all infants. This ratio is an indirect method of assessment and all such methods will have confounding variables. The relationship between TPV/ RVET and pulmonary artery pressure has been validated with direct measurements in several studies. ${ }^{9} 10$ The important documented confounders for TPV/RVET are heart rate, myocardial dysfunction, and pulsed Doppler sample position. ${ }^{11}$ The consistency of sample position was dealt with in the methods section and no infant had any evidence of myocardial dysfunction on two dimensional 
imaging. Heart rate is an important confounder in that faster heart rates have been described with the use of dexamethasone. ${ }^{6}$ Tachycardia will reduce TPV/RVET independently from changes in pulmonary artery pressure. However, longitudinal analysis of heart rate in this study revealed no change, suggesting this was not a factor in the changes in TPV/RVET which were observed.

Myocardial thickening has been previously described: Werner et al studied eight preterm infants and showed an average increase in EDIVS thickness of $1.4 \mathrm{~mm}$ and of EDLVPW thickness of $1.26 \mathrm{~mm} .{ }^{6}$ This current study found very similar mean increases in left ventricular myocardial thickness. However, two points emerge from these data which were not emphasised in the paper of Werner et al. Firstly that the myocardial thickening was not always generalised, while seven of the infants showed generalised thickening, two infants showed septal thickening with minimal posterior wall change and vice versa for two infants. Secondly and more importantly, that the statistical distribution of increase in particularly the septal thickness is not Gaussian, thus the median increase for EDIVS and EDLVPW was lower than the mean, respectively 0.9 and $0.8 \mathrm{~mm}$. In six of the infants the increase in septal thickness was small, less than $1 \mathrm{~mm}$. The other five demonstrated much more marked changes, two of them developing Doppler evidence of LVOT obstruction. Whether this was symptomatic in the most severely affected infant is difficult to prove. Clinical and radiological evidence together with the rapid response to diuretics suggested a cardiac cause for the deterioration. The LVOT gradient at that time was minimal but with the degree of hypertrophy and left ventricular chamber occlusion, there may have been a more generalised problem with left ventricular function. ${ }^{6}$ That dexamethasone induced myocardial thickening can become symptomatic is also supported by the report of Brand et al, ${ }^{7}$ which describes three preterm infants where LVOT obstruction together with symptoms of cardiac failure developed during dexamethasone treatment. It would seem prudent to suggest that preterms receiving steroids should have echocardiography in the second week of treatment and that those with significant myocardial thickening should be followed up to monitor this side effect. The action to be recommended when severe myocardial hypertrophy occurs will depend on the respiratory status of the infant. Most of the respiratory improvement associated with the use of steroids occurs in the first week of treatment. Hypertrophy resolves very quickly once steroids are stopped so it may be prudent to shorten the course of dexamethasone when significant hypertrophy is documented. Why myocardial thickening occurs remains unclear. Part of the cause may be the increase in systemic blood pressure which has been documented with the use of steroids. Blood pressure was not formally monitored in this study, however, increases similar to that described by Werner et al ${ }^{6}$ were seen in the few infants still having continuous intra-arterial monitoring when steroids were given. Against this being the sole explanation is the fact that left ventricular hypertrophy is not always generalised and that right ventricular thickening also occurs, not studied by us but described by Werner et al. Also the speed with which the problem resolves would suggest that reactive hypertrophy of heart muscle is not the sole explanation. In the most severe case, in this study, the EDIVS thickness reduced by $2.6 \mathrm{~mm}$ in the four days after steroids were stopped. Animal studies have suggested that direct cellular effects of steroids on the heart muscle may have a role. ${ }^{18}$

It has also been hypothesised that steroids augment closure of a patent ductus arteriosus. Animal studies have shown that steroids potentiate the effect of indomethacin in inducing ductal constriction. ${ }^{19}$ Heyman et al described four preterm infants in whom there was a temporal association between the commencing of dexamethasone and the disappearance of a ductal murmur. ${ }^{8}$ In only one of these cases was both the diagnosis and closure confirmed on echocardiography. Of the four infants in this study with echocardiographic evidence of a patent ductus arteriosus when steroids commenced, only one closed in the first week of treatment and one did not close at all. The lack of relationship between duct closure and steroids commencing and also the fact that closure was occurring at a postnatal age when spontaneous late closure would be common, would suggest that dexamethasone has no consistent effect on ductal closure in this situation.

In conclusion, this study found no evidence to support either the hypothesis that steroids may improve chronic lung disease through a direct effect on pulmonary vascular resistance or the hypothesis that they may augment ductal closure. Steroid induced myocardial hypertrophy, in preterm infants, is usually mild but can be severe and potentially symptomatic. It would seem prudent that preterm infants receiving steroids should be monitored echocardiographically for this side effect.

1 Yoder MC, Chua R, Tepper R. Effect of dexamethasone on pulmonary inflammation and pulmonary function of ventilator dependent infants with bronchopulmonary dysplasia. Am Rev Respir Dis 1991; 143: 1044-8.

2 Kuehl FA, Egan RW. Prostaglandins, arachidonic acid and inflammation. Science 1980; 210: 978-83.

3 Flower RJ, Blackwell GJ. Anti-inflammatory steroids induce biosynthesis of a phospholipase $\mathrm{A}_{2}$ inhibitor which prevents prostaglandin generation. Nature 1979; 278 456-9.

4 Philips JB, Lyrene RK. Prostaglandins, related compounds and the perinatal circulation. Clin Perinatol 1984; 11: 565-79.

5 Evans NJ, Archer LNJ. Doppler assessment of pulmonary artery pressure during the recovery period of hyaline membrane disease. Arch Dis Child 1991; 66: 802-4.

6 Werner JC, Sicard RE, Hansen R, Solomon E, Cowett RM, Oh W. Hypertrophic cardiomyopathy associated with dexamethasone therapy for bronchopulmonary dysplasia. I Pediatr 1992; 120: $286-91$.

7 Brand P, Van Lingen RA, Brus F, Talsma MD, Elzenga NJ. Hypertrophic obstructive cardiomyopathy as a side effect of dexamethasone treatment for bronchopulmonary dy dexamethasone treatment for bronchopulr

8 Heyman E, Ohlsson A, Shennan AT, Heilbut M, Coceani F. Closure of patent ductus arteriosus after treatment F. Closure of patent ductus arteriosus after treatment
with dexamethasone. Acta Paediatr Scand 1990; 79: with dexam 
9 Kitabatake A, Inoue $\mathrm{M}$, Asao $\mathrm{M}$, et al. Non-invasive evaluation of pulmonary hypertension by a pulsed Dopple tion of pulmonary hypertension by a
technique. Circulation 1983; 68: 302-9.

10 Kosturakis D, Golberg SJ, Allen HD, Loeber C. Doppler echocardiographic prediction of pulmonary arterial pressure in congenital heart disease. Am f Cardiol 1984 53: $1110-5$.

11 Paniidis IP, Ross J, Mintz GS. Effect of sampling site on assessment of pulmonary artery blood flow by Doppler echocardiography. Am $\mathcal{F}$ Cardiol 1986; 58: 1145-7.

12 Sahn DJ, DeMaria A, Kisslo J, Weyman A Recommendations regarding quantitation in $M$-mode echocardiography: results of a survey of echocardiographic measurements. Circulation 1978; 58: 1072-83.

13 Evans NJ, Archer LNJ. Postnatal circulatory adaptation in healthy term and preterm neonates. Arch Dis Child 1990; 65: 24-6.

14 Cummins JJ, D'Eugenio DB, Gross SJ. A controlled trial of dexamethasone in preterm infants at high risk for bronchopulmonary dysplasia. $N$ Engl f Med 1989; 320: 1505-10.

15 Avery GB, Fletcher AB, Kaplan M, Brudno DS. Controlled trial of dexamethasone in respirator dependent infants with bronchopulmonary dysplasia. Pediatrics 1985; 75: 106-11.

16 Skinner JR, Boys RJ, Hunter S, Hey EN. Non-invasive assessment of pulmonary arterial pressure in healthy neonates. Arch Dis Child 1991; 66: 386-90.

17 Serwer GA, Cougle AG, Eckherd JM, Armstrong BE. Factors affecting use of the Doppler determined time from flow onset to maximal pulmonary artery velocity for measurement of pulmonary artery pressure in children. Am 7 Cardiol 1986; 58: 352-6.

18 Sicard RE, Werner JC. Dexamethasone induces a transient relative cardiomegaly in neonatal rats. Pediatr Res 1992; 31: 359-63.

19 Momma K, Takeo A. Increased constriction of the ductus arteriosus with combined administration of indomethacin and betamethasone in fetal rats. Pediatr Res 1989; 25: 69-75. 